\title{
ON DOUBLE-PULSE STABILITY CRITERIA WITH DAMPING*
}

\author{
BY \\ M. MORDUCHOW AND L. GALOWIN \\ Polytechnic Institute of Brooklyn
}

Summary. A simple necessary condition for the stability of a linear dynamic system with elastic and damping characteristics which vary periodically with the same period is derived. General explicit necessary and sufficient conditions for stability are then developed for a double-pulse system. Such a system can be characterized by a pair of eigenvalues, or complex frequencies, corresponding to each half-period, and the stability of this system depends only on these complex frequencies. It is shown that a necessary, though not sufficient, condition for the stability of any such system is that the arithmetic mean of the real parts of all four of the complex frequencies over an entire period be negative or zero. This is shown to be true, more generally, for an $n$-pulse system. The physical significance of the results is discussed, and numerical examples are given.

Introduction. A variety of physical phenomena ${ }^{1}$ are characterized by a linear homogeneous differential equation with periodic coefficients. Sometimes the chief interest is in the stability of the solutions of such equations, rather than in the solutions themselves. If the system which these equations characterize is such that the coefficients vary (periodically) by changing discontinuously from one constant value to another one or

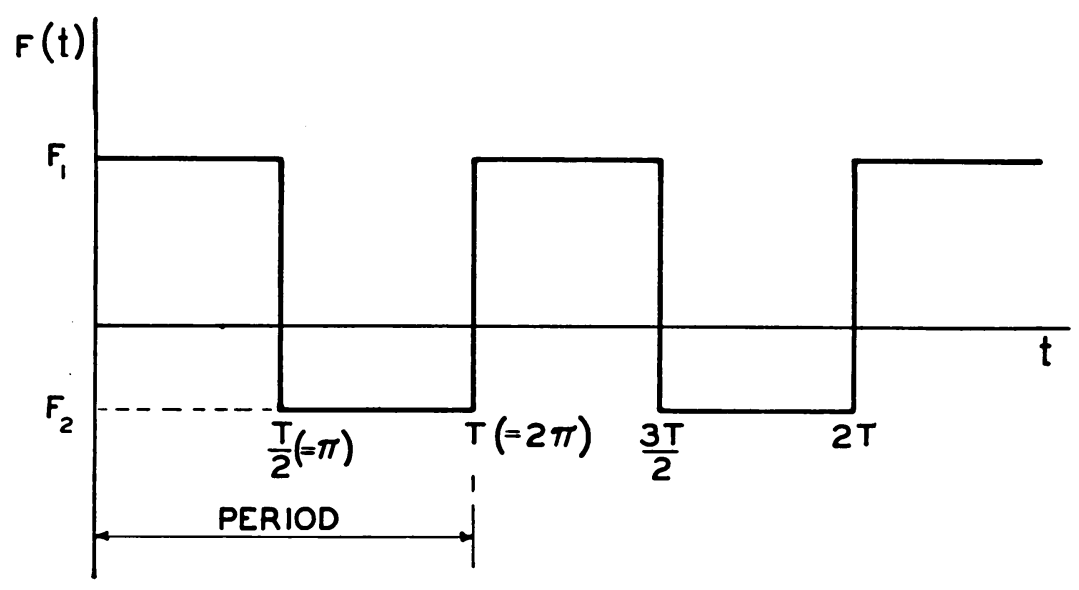

Fig. 1. Double-Pulse Function $F(t)$ with Period $T(=2 \pi)$.

more times during a period of variation (Figs. 1 and 2) then an exact solution of the equations can in principle be obtained without difficulty. Coefficients varying in the manner of Figs. 1 and 2 are called "rectangular pulses". In particular, if the coefficient

*Received March 29, 1951.

${ }^{1}$ Examples are given in: (a) B. Van der Pol \& M. J. O. Strutt, On the stability of the solutions of Mathieu's equation, Phil. Mag., 5, 18 (1928); (b) S. Timoshenko, Vibration problems in engineering, second edition, D. Van Nostrand Co., N. Y., 1937, Chapter III; (c) J. P. Den Hartog, Mechanical vibrations, third edition, McGraw-Hill Book Co., N. Y., 1947, p. 408. 
assumes only two different constant values, one for each half-period of variation (Fig. 1) it is called a "double-pulse". In cases in which the periodic coefficients do not vary as rectangular pulses, it is often possible to obtain approximate or at least qualitative solutions by replacing these coefficients by either double pulses or four pulses. ${ }^{2}$ This type of approximation has, in fact, been recently applied. ${ }^{3}$

The purpose of this paper is to present explicit stability criteria for systems which can be represented by equations with periodic, especially double-pulse, coefficients for

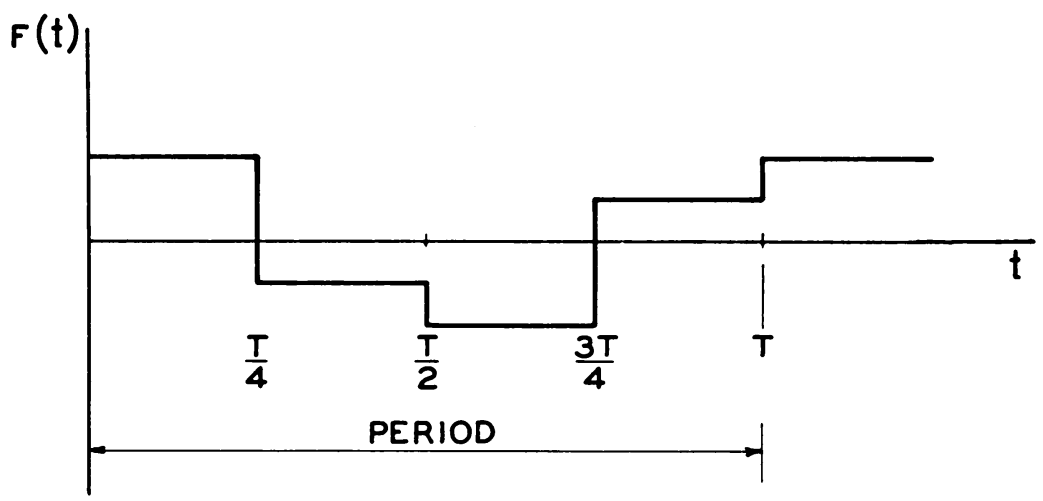

Fig. 2. Four-Pulse Function $F(t)$ with Period $T$.

the damping as well as for the elastic characteristics. Such a system without damping has been analyzed in detail by Meissner ${ }^{4}$ and by Van der Pol and Strutt. ${ }^{5}$ Schwerin $^{6}$ subsequently extended this investigation to systems with linearly varying characteristics. A particular double-pulse system with damping has also been investigated, ${ }^{7}$ without, however, attempts to derive explicit equations and conclusions for damped systems in general.

The results obtained in the present analysis serve to present useful theorems of physical interest, to demonstrate some significant conclusions which might not be readily foreseen without the mathematical analysis, and to facilitate any actual stability calculations based on the double-pulse method.

General theory. The free motion of a linear dynamic system with spring stiffness $k$ and damping coefficient $f$ varying periodically with time, with period $2 \pi$, can be represented by the equation:

$$
x^{\prime \prime}+f(t) x^{\prime}+k(t) x=0
$$

${ }^{2}$ B. Van der Pol and M. J. O. Strutt, op. cit.

${ }^{3} \mathrm{G}$. Horvay and S. W. Yuan, Stability of rotor blade flapping motion when the hinges are tilted. Generalization of the "rectangular ripple" method of solution, J. Aero. Sci., 14, 583-593, (1947). Also, S. W. Yuan and M. Morduchow, On the stability of the transient motion of helicopter blades in flapping and lagging, Reissner Anniv. Vol., J. W. Edwards, 1949, p. 163.

${ }^{4}$ E. Meissner, Über Schüttelschwingungen in Systemen periodisch veränderlicher Elastizität, Schweizer. Bauzeitung, 1918, p. 95 (1918).

5op. cit.

${ }^{6} \mathrm{E}$. Schwerin, Ein allgemeines Integrationsverfahren für quasiharmonische Schwingungsvorgänge, Z. Techn. Phys. 12, 104 (1931).

${ }^{7}$ G. Horvay and S. W. Yuan, op. cit. Also, S. W. Yuan and M. Morduchow, op. cit. 
where $x(t)$ denotes displacement, the primes denote differentiation with respect to the time $t$, and $f(t)$ and $k(t)$ are functions having the period $2 \pi$. By letting

$$
x=v(t) \exp \left(-\frac{1}{2} \int f d t\right)
$$

Eq. (1) can be transformed into the form

$$
v^{\prime \prime}+k_{1}(t) v=0,
$$

where $k_{1}(t)$ has the period $2 \pi$. By Floquet's theory ${ }^{8}$ at least one particular integral of Eq. (3) is of the form

$$
v_{1}=\phi(t) \exp (\mu t)
$$

where $\phi(t)$ is a function with the period $2 \pi$, and $\mu$ is a definite constant. From this it can be shown that a second independent integral of Eq. (3) must have the form

$$
v_{2}=\psi(t) \exp (-\mu t)
$$

where $\psi(t)$ has the period $2 \pi$.

From Eq. (2), and from the fact that, due to the periodicity of $f(t)$,

$$
\int_{t}^{t+2 \pi} f(t) d t=\text { constant }=C,
$$

say, it follows that the solutions $x_{1}(t)$ and $x_{2}(t)$ of Eq. (1), corresponding to $v_{1}$ and $v_{2}$ respectively, will satisfy the following relations, regardless of the time $t$ :

$$
x_{1}(t+2 \pi) / x_{1}(t)=\sigma_{1}, \quad x_{2}(t+2 \pi) / x_{2}(t)=\sigma_{2}
$$

where $\sigma_{1}$ and $\sigma_{2}$ are constants given by

$$
\sigma_{1}=\exp \left(2 \pi \mu-\frac{1}{2} C\right), \quad \sigma_{2}=\exp \left(-2 \pi \mu-\frac{1}{2} C\right) .
$$

The general solution of Eq. (1) can be written as

$$
x=A_{1} x_{1}(t)+A_{2} x_{2}(t),
$$

where $A_{1}$ and $A_{2}$ are arbitrary constants.

The dynamic system may be defined as stable when both of its component free motions $x_{1}$ and $x_{2}$ subside with time, and unstable if either of these motions amplifies with time. From Eqs. (6a) it then follows that the system will be stable if $\left|\sigma_{1}\right|<1$ and $\left|\sigma_{2}\right|<1$, while the system will be unstable if either $\left|\sigma_{1}\right|>1$ or $\left|\sigma_{2}\right|>1$. The case $\left|\sigma_{1}\right|=1$ and $\left|\sigma_{2}\right|=1$ may be defined as one of neutral stability.

Equations (6b) imply that $\sigma_{1} \sigma_{2}=\exp (-C)$. Hence in order that both $\left|\sigma_{1}\right| \leq 1$ and $\left|\sigma_{2}\right| \leq 1$, it is necessary that $C \geq 0$. This can be expressed by the following theorem:

Theorem I: A necessary condition for the stability of a dynamic system characterized by an equation of the form (1) is that

$$
\int_{0}^{2 \pi} f(t) d t \geq 0
$$

${ }^{8} \mathrm{M}$. G. Floquet, Sur les équations différentielles linéaires à coefficients périodiques, Ann. Ec. Norm. 12, 47 (1883). Also, E. T. Whittaker and G. N. Watson, Modern analysis, Macmillan Co., N. Y., 1948, pp. 412-413. 
Physically this condition can be interpreted as requiring that the average value of the periodic damping coefficient $f(t)$ during any period must be non-negative. Thus, for stability, there must in the average be a positive damping force.

Double-pulse system. To investigate, in particular, the stability of a double-pulse system with damping, let $f(t)$ and $k(t)$ vary periodically in the manner of Fig. 1, i.e. let $f=f_{1}$ and $k=k_{1}$ for $0<t<\pi$, while $f=f_{2}$ and $k=k_{2}$ for $\pi<t<2 \pi$; the constants $f_{1}, f_{2}, k_{1}$ and $k_{2}$ may be positive or negative. Then Eq. (1) can be solved for each of the two time-intervals as a linear equation with constant coefficients. The solution can be expressed as:

$$
\begin{array}{ll}
x=C_{1} \exp \left(p_{1} t\right)+\bar{C}_{1} \exp \left(\bar{p}_{1} t\right), & 0 \leq t \leq \pi \\
x=C_{2} \exp \left(p_{2} t\right)+\bar{C}_{2} \exp \left(\bar{p}_{2} t\right), & \pi \leq t \leq 2 \pi
\end{array}
$$

where the $C_{i}$ and $\bar{C}_{i}$ are arbitrary constants, the $p_{i}$ and $\bar{p}_{i}$ are constants given by:

$$
p_{i}=-\frac{f_{i}}{2}+\left[\left(\frac{f_{i}}{2}\right)^{2}-k_{i}\right]^{1 / 2}, \quad \bar{p}_{i}=-\frac{f_{i}}{2}-\left[\left(\frac{f_{i}}{2}\right)^{2}-k_{i}\right]^{1 / 2}
$$

The $p_{i}$ and $\bar{p}_{i}$ are essentially eigenvalues corresponding to each half-period of variation of the damping and elastic coefficients, and are sometimes called "complex frequencies". Their physical interpretation follows from Eqs. (8). Thus, if a system were characterized by constant dynamical coefficients leading to a (single) pair of complex conjugate values for $p$ and $\bar{p}$, then the system would oscillate freely with a natural frequency proportional to the imaginary part of $p$ or $\bar{p}$, and would be damped out with a logarithmic decrement directly proportional to the negative of the real part and inversely proportional to the imaginary part of $p$ or $\bar{p}$. A system with constant coefficients characterized by a single pair of real values of $p$ and $\bar{p}$ would be non-oscillatory and would be stable if both $p$ and $\bar{p}$ are negative, but unstable if either $p$ or $\bar{p}$ is positive. For convenience, the negatives of the real parts of $p_{i}$ and $\bar{p}_{i}$ will here be called "damping factors".

The arbitrary constants $C_{i}$ and $\bar{C}_{i}$ in Eqs. (8) are related by the two conditions of continuity of displacement $x$ and velocity $\dot{x}$ at $t=\pi$. Moreover, the following two additional conditions can be prescribed:

$$
(x)_{t=2 \pi}=\sigma(x)_{t=0}, \quad\left(x^{\prime}\right)_{t=2 \pi}=\sigma\left(x^{\prime}\right)_{t=0},
$$

where $\sigma$ is a constant, to be determined. It can be shown from Eqs. (2), (4a, b) and (7) that by satisfying conditions (10), initial conditions are implicitly prescribed so that $A_{2}$ or $A_{1}$ in Eq. (7) will be zero, ${ }^{9}$ and so that consequently the two values of $\sigma$ which will thus be obtained will correspond exactly to $\sigma_{1}$ and $\sigma_{2}$ respectively of Eqs. (6b). By applying the continuity conditions and conditions (10) to Eqs. (8) a set of four linear homogeneous equations in $C_{1}, \bar{C}_{1}, C_{2}$ and $\bar{C}_{2}$ is obtained. The condition for the existence of a non-trivial solution to these equations leads to a determinantal equation, which when expanded can be written in the form:

$$
\sigma^{2}+N \sigma+M=0
$$

where

'This fact, although implied, often appears not to be clearly stated in the literature. 


$$
\begin{gathered}
N=\frac{\left(p_{1}-\bar{p}_{2}\right)\left(p_{2}-p_{1}\right)\left(P_{1} \bar{P}_{2}+\bar{P}_{1} P_{2}\right)+\left(p_{2}-\bar{p}_{1}\right)\left(\bar{p}_{2}-p_{1}\right)\left(P_{1} P_{2}+\bar{P}_{1} \bar{P}_{2}\right)}{\left(\bar{p}_{1}-p_{1}\right)\left(\bar{p}_{2}-p_{2}\right)}, \\
M=P_{1} \bar{P}_{1} P_{2} \bar{P}_{2}, \\
P_{i}=\exp \left(p_{i} \pi\right), \quad \bar{P}_{i}=\exp \left(\bar{p}_{i} \pi\right) .
\end{gathered}
$$

The roots

$$
\sigma=-\frac{N}{2} \pm\left[\left(\frac{N}{2}\right)^{2}-M\right]^{1 / 2}
$$

of Eq. (11) then determine the stability of the system.

Three general cases of double-pulse systems, depending on whether the eigenvalues $p_{i}$ and $\bar{p}_{i}$ are real or complex, can be distinguished. By substitution into Eqs. (12a, b) the following expressions for $N$ and $M$ in these various cases are obtained $\left(a_{i}, b_{i}, c_{i}\right.$ and $d_{i}$ denote real positive or negative constants, while $\left.i=(-1)^{1 / 2}\right)$.

CASE I. Two pairs of complex conjugate eigenvalues:

$$
\begin{gathered}
p_{1}=-a_{1}+b_{1} i, \quad \bar{p}_{1}=-a_{1}-b_{1} i, \quad p_{2}=-a_{2}+b_{2} i, \quad \bar{p}_{2}=-a_{2}-b_{2} i . \\
2 b_{1} b_{2} N=\left(\exp \left[-\pi\left(a_{1}+a_{2}\right)\right]\right)\left\{\left[\left(a_{1}-a_{2}\right)^{2}+\left(b_{1}-b_{2}\right)^{2}\right] \cos \left(b_{1}-b_{2}\right) \pi\right. \\
\left.-\left[\left(a_{1}-a_{2}\right)^{2}+\left(b_{1}+b_{2}\right)^{2}\right] \cos \left(b_{1}+b_{2}\right) \pi\right\} \\
M=\exp \left[-2 \pi\left(a_{1}+a_{2}\right)\right]
\end{gathered}
$$

CASE II. Two pairs of real eigenvalues:

$$
\begin{gathered}
p_{1}=-c_{1}, \quad \bar{p}_{1}=-d_{1}, \quad p_{2}=-c_{2}, \quad \bar{p}_{2}=-d_{2} . \\
\left(c_{1}-d_{1}\right)\left(c_{2}-d_{2}\right) N=\left(d_{1}-d_{2}\right)\left(c_{2}-c_{1}\right)\left\{\exp \left[-\pi\left(c_{1}+d_{2}\right)\right]\right. \\
\left.+\exp \left[-\pi\left(c_{2}+d_{1}\right)\right]\right\} \\
+\left(c_{2}-d_{1}\right)\left(d_{2}-c_{1}\right)\left\{\exp \left[-\pi\left(c_{1}+c_{2}\right)\right]+\exp \left[-\pi\left(d_{1}+d_{2}\right)\right]\right\} \\
M=\exp \left[-\pi\left(c_{1}+d_{1}+c_{2}+d_{2}\right)\right]
\end{gathered}
$$

CASE III. One pair of complex conjugate, and one pair of real eigenvalues:

$$
p_{1}=-a_{1}+b_{1} i, \quad \bar{p}_{1}=-a_{1}-b_{1} i, \quad p_{2}=-c_{2}, \quad \bar{p}_{2}=-d_{2}
$$

$$
\begin{aligned}
& b_{1}\left(d_{2}-c_{2}\right) N \\
& =\left(\exp \left[-\pi a_{1}\right]\right)\left\{\left[\left(d_{2}-a_{1}\right)\left(a_{1}-c_{2}\right)-b_{1}^{2}\right]\left[\exp \left(-\pi d_{2}\right)-\exp \left(-\pi c_{2}\right)\right] \sin b_{1} \pi\right. \\
& \left.+b_{1}\left(c_{2}-d_{2}\right)\left[\exp \left(-\pi d_{2}\right)+\exp \left(-\pi c_{2}\right)\right] \cos b_{1} \pi\right\} \\
& M=\exp \left[-\pi\left(2 a_{1}+c_{2}+d_{2}\right)\right]
\end{aligned}
$$


For any given double-pulse system, the stability characteristics can be quickly and straight-forwardly determined by calculating the $p_{i}$ and $\bar{p}_{i}$ from Eqs. (9), evaluating $N$ and $M$ by means of the appropriate pair of expressions from the set $(14 \mathrm{a}, \mathrm{b}),(15 \mathrm{a}, \mathrm{b})$ and $(16 \mathrm{a}, \mathrm{b})$, and then obtaining the two values of $\sigma$ from Eq. (13). It should be noted that these calculations will always involve only real quantities.

From Theorem I it follows that for stability of a double-pulse system it is necessary that $\left(f_{1}+f_{2}\right) \geq 0$. According to Eqs. (9), this is equivalent to the following theorem: ${ }^{10}$

Theorem II: If a double-pulse system is characterized in one half-period by eigenvalues $p_{1}$ and $\bar{p}_{1}$ and in the other half-period by eigenvalues $p_{2}$ and $\bar{p}_{2}$, then a necessary condition that the system be stable is that the arithmetic mean of the real parts of $p_{1}, \bar{p}_{1}, p_{2}$ and $\bar{p}_{2}$ be negative or zero. Alternatively, a sufficient condition that the system be unstable is that the arithmetic mean of the real parts of $p_{1}, \bar{p}_{1}, p_{2}$ and $\bar{p}_{2}$ be positive.

By virtue of Theorem I and of relations of the form (9) it can be quite similarly proven that Theorem II is valid more generally for an $n$-pulse system. Moreover, it similarly follows that if during any period of an $n$-pulse system, $f(t)$ assumes constant values $f_{1}, f_{2}, \cdots, f_{n}$ during unequal time-intervals, then a necessary condition for stability is that the weighted mean of the real parts of the complex frequencies $p_{1}$ to $p_{n}$ be negative or zero, the weights being proportional to the time-intervals corresponding to each of the $p$ 's.

It may be noted that the above necessary condition for the stability of a doublepulse system is in a sense less stringent than that of a system with constant elastic and damping coefficients, since in the latter case it would be required that each damping factor be positive, and not merely the average of the damping factors. It must be observed, on the other hand, that the theorems derived here give only necessary, but not sufficient, conditions for stability. This follows essentially from the fact that although the conditions $\left|\sigma_{1}\right| \leq 1,\left|\sigma_{2}\right| \leq 1$ always imply $\sigma_{1} \sigma_{2} \leq 1$, the converse is not necessarily true. As a consequence, a double-pulse system may be unstable even under conditions for which a constant-coefficient system would be stable. For example, it is well known that in the absence of damping forces a constant-coefficient system will be neutrally stable when the restoring force is positive $(k>0)$, leading to purely imaginary complex frequencies. However, as can be verified by putting $a_{1}=a_{2}=0$ in Eqs. (14a, b) and noting that values of $b_{1}$ and $b_{2}$ (e.g. $b_{1}=1.5, b_{2}=0.5$ ) exist for which $|N|>2$ and hence $|\sigma|>1$, a double-pulse system may in such a case be unstable.

From Eqs. (14) and (16) in conjunction with (13) it is seen that although the necessary condition for stability stated here involves only the damping factors, the necessary and sufficient condition involves the natural frequencies as well. This is partly in contrast to a system with constant damping and spring characteristics, where the stability depends exclusively on the damping factors. Considering, for example, Case I, which is in practice the most important case, it can be seen from Eqs. $(14 \mathrm{a}, \mathrm{b})$ that a large positive value of $\left(a_{1}+a_{2}\right)$ tends to keep the values of $|N|$ and $|M|$ far below unity, and hence strongly tends to make the system stable. This result appears quite reasonable on physical grounds. Nevertheless, it will be found that for any given value of $\left(a_{1}+a_{2}\right)$, i.e. for any given amount of damping in the average, there will exist values of the fre-

${ }^{10}$ The theorem which follows could also be directly derived by noting that for stability it is necessary that $|M| \leq 1$, and by using the expressions (14b), (15b) and (16b) for $M$. 
quency ratio $b_{1} / b_{2}$ for which the system will be unstable. This can be verified by observing that for any given positive value of $\left(a_{1}+a_{2}\right)$, however large, sufficiently large values of $b_{1} / b_{2}\left(\right.$ or $\left.b_{2} / b_{1}\right)$ can be found for which $|N|$ as given by Eq. (14a) will be large enough to make at least one of the roots in Eq. (13) greater than unity in absolute value.

\section{Examples}

1. As an example illustrating the insufficiency of the necessary condition derived here for stability, suppose that in Case I $a_{1}=5, a_{2}=-4$ and $b_{1}=b_{2}=1.5$. Then the average of the damping factors $a_{1}$ and $a_{2}$ is here positive; nevertheless this system is unstable, for Eqs. (14a) and (14b) yield: $N=38.0$ exp $(-\pi), M=\exp (-2 \pi)$. The larger of the roots of Eq. (13) will be $|\sigma|=1.64$, which exceeds 1 and hence implies instability.

2. The purpose of the second example is to show that a system pertaining to Case II can, contrary to systems with constant characteristics, be stable even when the average of a single pair of damping factors over a period, e.g. $\left(c_{1}+d_{2}\right) / 2$ or $\left(c_{1}+c_{2}\right) / 2$, is negative. Thus, let $c_{1}=1, c_{2}=-1.333, d_{1}=1.333$ and $d_{2}=0.880$. Then $\left(c_{1}+c_{2}\right)<0$. Eqs. (15a, b) however give: $N=-0.209, M=0.00169$. The roots (13) for $\sigma$ are $\sigma_{1}=$ 0.201 and $\sigma_{2}=0.0081$, and the system is therefore stable.

It may be noted that in either of the above two numerical examples, it might have been difficult to predict in advance the stability or instability of the given system.

\section{ConcLusions}

1. A necessary condition for the stability of a linear dynamic system with damping coefficient $f(t)$ and elastic coefficient both varying periodically with the period $2 \pi$ is

$$
\int_{0}^{2 \pi} f(t) d t \geq 0 .
$$

2. In particular, a necessary condition for the stability of an $n$-pulse system is that the weighted arithmetic mean of the real parts of the $2 n$ "complex frequencies" characterizing the system during any period of the variation of the damping and elastic coefficients be negative or zero. The weights must be proportional to the time-intervals, of any period, corresponding to each of the complex frequencies. For equal time-intervals, of course, the simple arithmetic mean is taken. The case $n=2$ with equal time-intervals ("double-pulse"), was of especial concern here.

3. Contrary to systems with constant damping and elastic coefficients, the necessary and sufficient condition for the stability of a double-pulse system depends, in general, not only on the damping factors, or (negative) real parts of the complex frequencies, but also on the natural frequencies, or imaginary parts of the complex frequencies. Moreover, contrary to a system with constant coefficients, a double-pulse system can be stable even when not each of the damping factors is positive or zero.

4. The stability characteristics of any given double-pulse system with (positive and/or negative) damping can in practice be quickly calculated from the equations developed here.

The authors hereby express their thanks to Professor R. M. Foster for his valuable suggestions and comments. 